\section{Prospective Comparison of Gd-EOB-DTPA- Enhanced MRI with Dynamic CT for Detecting Recurrence of HCC after Radiofrequency Ablation}

\author{
Yasuharu Imai $^{a}$ Kazuhiro Katayama $^{b}$ Masatoshi Hori ${ }^{c}$ Takayuki Yakushijin ${ }^{d}$ \\ Kenji Fujimoto $^{e}{ }^{f}$ Toshifumi Itoh $^{g}$ Takumi Igura $^{a}$ Mitsuru Sakakibara $^{b}$ \\ Manabu Takamurah ${ }^{h}$ Masakatsu Tsurusaki ${ }^{i}$ Hiroto Takahashic \\ Katsuyuki Nakanishi ${ }^{j}$ Noriaki Usuki ${ }^{k}$ Koh Tsuji Hiroshi Ohashim ${ }^{\mathrm{m}}$ Tonsok Kim $^{\mathrm{c}}$

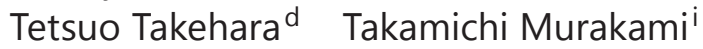 \\ a Department of Gastroenterology, Ikeda Municipal Hospital, Ikeda, ${ }^{b}$ Department of Hepatobiliary \\ and Pancreatic Oncology, Osaka International Cancer Institute, Osaka, Departments of ${ }^{c}$ Radiology \\ and ${ }^{\mathrm{d}}$ Gastroenterology and Hepatology, Osaka University Graduate School of Medicine, \\ Suita, ${ }^{e}$ Department of Internal Medicine and ${ }^{\mathrm{f}}$ Division of Clinical Research, National Hospital \\ Organization Minamiwakayama Medical Center, Tanabe, ${ }^{9}$ Department of Gastroenterology and \\ Hepatology, JCHO Osaka Hospital, Osaka, h Department of Radiology, Ikeda Municipal Hospital, \\ Ikeda, 'Department of Radiology, Faculty of Medicine, Kindai University, Sayama, ${ }^{j}$ Department \\ of Diagnostic Radiology, Osaka International Cancer Institute, and ' Department of Radiology, \\ JCHO Osaka Hospital, Osaka, 'Department of Radiology, National Hospital Organization \\ Minamiwakayama Medical Center, Tanabe, and ${ }^{\mathrm{m}}$ Department of Pathology, Ikeda Municipal \\ Hospital, Ikeda, Japan
}

\section{Keywords}

Hepatocellular carcinoma - Radiofrequency ablation · Gd-EOB-DTPA-MRI · Computed tomography $\cdot$ Recurrence

\begin{abstract}
Background: We prospectively compared the efficacy of gadolinium-ethoxybenzyl-diethylenetriamine pentaacetic acid (Gd-EOB-DTPA)-enhanced magnetic resonance imaging (MRI) with that of dynamic multidetector computed tomography (MDCT) for detection of recurrent hypervascular hepatocellular carcinoma (HCC) after radiofrequency ablation (RFA). Methods: Institutional review board approval and written informed consent were obtained for this multicenter study. Ninety-seven HCC patients treated with curative RFA underwent both Gd-EOBDTPA-enhanced MRI and dynamic MDCT every 3-4 months. HCC diagnosis was made based on the typical enhancement pattern of HCC on MRI and/or CT by on-site consensus reading. Two blinded observers independently assessed image datasets to compare diagnostic accuracy, sensitivity, specificity, and the areas under the receiver operating characteristic curve

Yasuharu Imai, MD, PhD

Department of Gastroenterology, Ikeda Municipal Hospital

3-1-18, Johnan, Ikeda

Osaka 563-8510 (Japan)

E-Mail yasuimai@hosp.ikeda.osaka.jp
\end{abstract}


(AUROC). Results: Recurrence was observed in 48 of 97 patients. Among these, 22 were diagnosed by both Gd-EOB-DTPA-enhanced MRI and MDCT; the remainder were diagnosed by only one of these 2 modalities. Recurrence was diagnosed in more patients by Gd-EOB-DTPAenhanced MRI than by MDCT (44 vs. 26 patients, $p<0.001$ ). Patient-based analysis revealed that the accuracy, sensitivity, and AUROC of Gd-EOB-DTPA-enhanced MRI were significantly higher than those of MDCT for both observers $(p<0.005)$. The AUROC of Gd-EOB-DTPAenhanced MRI and MDCT was 0.95 and 0.76 for observer 1 and 0.90 and 0.74 for observer 2, respectively. The $\mathrm{K}$ values for MRI and MDCT were 0.83 and 0.70 , respectively. Conclusions: Compared with dynamic MDCT, Gd-EOB-DTPA-enhanced MRI had higher diagnostic accuracy and sensitivity for detection of recurrent hypervascular HCC and may be a better tool for following patients after RFA.

(C) 2017 S. Karger AG, Basel

\section{Introduction}

The global burden of cancer continues to increase owing to the aging and growth of the world population. Hepatocellular carcinoma (HCC) is one of the most common cancers worldwide, ranked fifth in men and seventh in women [1]. HCC is most prevalent in Asia and Africa, but it continues to increase in Western countries [2, 3]. Surveillance for HCC in highrisk groups has increased opportunities for curative treatment owing to early detection [4-6]. However, the recurrence rate of HCC after curative treatment is still high, mainly because of its multicentric recurrence behavior [7, 8]. Accordingly, an effective follow-up program for early and accurate detection of recurrence of HCC after curative treatment, including resection and radiofrequency ablation (RFA) therapy, is needed.

Gadolinium-ethoxybenzyl-diethylenetriamine pentaacetic acid (Gd-EOB-DTPA) (Bayer Yakuhin, Ltd., Berlin, Germany) is a magnetic resonance imaging (MRI) contrast agent, which in addition to enhancing the blood, has hepatocyte-specific properties. Focally decreased enhancement in the hepatobiliary phase of Gd-EOB-DTPA-enhanced MRI in the early stage of multistep hepatocarcinogenesis can signal early HCC [9-12]. Gd-EOB-DTPA-enhanced MRI has been reported to have higher diagnostic accuracy and sensitivity compared with dynamic computed tomography (CT) and now plays an important role in the detection of HCCs [1319]. Recent studies have reported that Gd-EOB-DTPA-enhanced MRI as an additional imaging modality to dynamic multidetector CT (MDCT) led to the detection of additional intrahepatic HCC lesions in patients with Barcelona Clinic Liver Cancer (BCLC) stage 0 or A HCC and subsequent modification of treatment plans, which brought about improved recurrence-free and overall survival [20-21].

The purpose of this study was to compare prospectively the efficacy of Gd-EOB-DTPAenhanced MRI with that of dynamic MDCT in the detection of intrahepatic recurrence of hypervascular HCC following curative treatment of HCC by RFA.

\section{Materials and Methods}

Patients

We enrolled patients from November 2010 through December 2012 at 5 hospitals. The inclusion criteria were: (1) 20 years of age or older; (2) body weight $\leq 100 \mathrm{~kg}$; (3) any cause of underlying liver disease; (4) patients who had undergone curative RFA treatment for HCC with a typical vascular pattern (arterial hypervascularity and portal venous/equilibrium phase washout according to the guidelines of the American Association for the Study of Liver Diseases [5]); and (5) patients who received dynamic CT or Gd-EOB-DTPAenhanced MRI within 1 month after RFA for diagnosis of curative treatment. A total of 114 patients were 
Table 1. Baseline characteristics of 97 patients

Mean age \pm SD (range), years

Gender (male/female)

Etiology (HBV-related/HCV-related/alcoholic/others)

Child-Pugh class (A/B)

Mean serum albumin \pm SD (range), $\mathrm{g} / \mathrm{dL}$

Mean total bilirubin \pm SD (range), $\mathrm{mg} / \mathrm{mL}$

Mean prothrombin time \pm SD (range), $\%$

Mean platelet count \pm SD (range), $\times 10^{3} / \mathrm{mL}$

Mean tumor size \pm SD (range), $\mathrm{mm}$

Tumor number (1/2/3/4)

\author{
$73 \pm 9(45-92)$ \\ $64 / 33$ \\ $12 / 73 / 5 / 7$ \\ $80 / 17$ \\ $3.6 \pm 0.5(2.6-4.8)$ \\ $0.9 \pm 0.4(0.4-2.6)$ \\ $81.9 \pm 14.0(39.9-120)$ \\ $12.0 \pm 4.9(3.9-23.7)$ \\ $13.6 \pm 5.8(5.0-34.0)$ \\ $72 / 21 / 3 / 1$
}

screened for enrollment. Of these patients, 9 were excluded for the following reasons: claustrophobia $(n=2)$, Child-Pugh grade C cirrhosis $(n=2)$, a history of anaphylactoid reaction to iodinated contrast media $(n=1)$, or receiving stereotactic body radiation therapy for HCC within 3 months $(n=4)$.

During the follow-up, 8 patients were dropped from the study within the first 6 months because of severe pain caused by bone metastasis $(n=1)$, cerebral bleeding $(n=1)$, development of impaired renal function $(n=1)$, retraction of consent $(n=2)$, or loss to follow-up $(n=3)$. Therefore, 97 patients were included for further analysis. The baseline characteristics of the 97 patients are presented in Table 1 . The median follow-up period was 385 days (range 86-1,141 days).

\section{Study Design}

Institutional review board approval and written informed consent from each patient were obtained at each participating hospital. This study was carried out in compliance with the Helsinki Declaration. It was registered under clinical trial No. UMIN000010887. The enrolled patients underwent both Gd-EOB-DTPAenhanced MRI and dynamic MDCT every 3-4 months and were followed until the end of December 2013. At each visit, the interval between Gd-EOB-DTPA-enhanced MRI and MDCT was within 3 weeks in random order. Diagnosis of recurrence of HCC was made on the basis of the typical vascular pattern of HCC of both Gd-EOB-DTPA-enhanced MRI and dynamic MDCT by consensus of a radiologist and a hepatologist with more than 5 years of experience in hepatobiliary imaging at each hospital (on-site consensus reading). Clinical information was available at the time of consensus reading. For Gd-EOB-DTPA-enhanced MRI, washout was defined as hypointensity in the portal venous phase, and the diagnosis of HCC was also made when the lesion showed arterial hypervascularity and hypointensity in the hepatobiliary phase or arterial hypervascularity, portal venous phase washout, and hyperintensity in hepatobiliary phase [16]. When only the MRI or CT showed the typical vascular pattern of HCC, contrast-enhanced ultrasonography (CEUS) or angiographyassisted CT, i.e., CT during hepatic arteriography (CTHA) and CT during arterial portography (CTAP), was additionally undertaken to confirm the diagnosis of HCC.

When the lesion was $\geq 1.5 \mathrm{~cm}$ and showed a hypovascular pattern (e.g., a lesion without arterial hypervascularity and with hypoattenuation/hypointensity in venous/late phase on multiphasic MDCT or the hepatobiliary phase on Gd-EOB-DTPA-enhanced MRI) a biopsy was performed as described previously [12], because these lesions were strongly suspected to be HCC [22]. Histological diagnosis of HCC was made as described previously by 1 pathologist with 17 years of experience [23]. When a histological diagnosis was HCC, the nodule was treated with RFA. When the histological diagnosis was not HCC, the patient continued to undergo both Gd-EOB-DTPA-enhanced MRI and dynamic MDCT every 3-4 months. These hypovascular HCC lesions were not included in the diagnostic performance analysis of this study because the study's purpose was to compare the efficacy of imaging techniques in the detection of intrahepatic recurrence of hypervascular HCC.

\section{Diagnostic Performance Analysis for Hypervascular HCC by Blinded Observers}

In addition to the on-site consensus reading, we conducted diagnostic performance analysis for hypervascular HCC with blinded observers. We gathered all MRI and CT datasets for independent review by 2 blinded observers. Diagnostic accuracy, sensitivities, specificities, and positive and negative predictive values were compared between Gd-EOB-DTPA-enhanced MRI and dynamic MDCT on patient-based and tumor-by-tumor analyses. 
For the 97 patients, regardless of HCC development during the follow-up period, dynamic MDCT and Gd-EOB-DTPA-enhanced dynamic MRI with hepatobiliary phase and T2-weighted images were evaluated independently and blindly by 2 radiologists (observers 1 and 2 with 6 and 14 years of experience, respectively, in hepatobiliary imaging) without any clinical information including imaging findings and final diagnosis. In patients in whom recurrence was not found during the observation period, CT and MR images at the time of 2 visits before the last visit were used for the diagnostic performance analysis because images at the last visit, which were obtained 6-8 months later, could be used as an evidence of no recurrence. The images were read on a picture archiving and communication system. During one session, image sets consisting of a mixture of MR images of half of the patients and CT images of the other half were presented in random order. During the other session, the remaining image sets were presented in random order. To minimize recall bias, each reading session was separated by at least a 4 -week interval.

Before the first reading session, the following criteria for diagnosing hypervascular HCC were provided to the observers: (a) hyperenhancement during the arterial phase; (b) hypoattenuation or hypointensity compared with the surrounding liver during the portal venous or equilibrium phases (only for CT) (i.e., washout); (c) hypointensity, isointensity, or hyperintensity during the hepatobiliary phase [16]. Hypervascular HCC was unequivocally diagnosed if a lesion fulfilled criterion (a) and any one of the other criteria (b-c). In addition, criteria for suspected but inconclusive diagnosis of hypervascular HCC included (d) mild hyperintensity on T2-weighted MRI with early enhancement or (e) nodular arterial enhancement without washout [16].

Each observer classified all detected lesions according to the following 4-point confidence score scale: 1 - probably no mass lesion present; 2 - indefinite presence of lesion; 3 - lesion probably present; and 4 definite presence of a lesion; with confidence scores of 3 and 4 representing a positive diagnosis of liver tumor.

\section{BCLC Stage at Recurrence}

We conducted a supplementary analysis to investigate BCLC stages at the time of recurrence and compared the stages diagnosed by Gd-EOB-DTPA-enhanced MRI and dynamic MDCT separately and together. In the BCLC stage analysis, both hypervascular and hypovascular HCCs diagnosed by using imaging and biopsy specimens were included because we thought that the data were important when describing the features of our subjects.

\section{Dynamic MDCT}

Dynamic MDCT was performed by using one of the four 64-channel MDCT scanners (Discovery CT $750 \mathrm{HD}^{\circledR}$, GE Healthcare, Milwaukee, WI, USA; Brilliance $64^{\circledR}$, Philips Medical Systems, Best, The Netherlands; Aquilion $\mathrm{CXL}^{\circledR}$ and Aquilion ONE ${ }^{\circledR}$, Toshiba Medical Systems, Tokyo, Japan). The tube voltage was $120-\mathrm{kVp}$ and the tube current was modulated automatically according to the patient's body size. The section thickness was $5 \mathrm{~mm}$. All patients were given $600 \mathrm{mg}$ of iodine per kilogram of body weight with 300 or 370 $\mathrm{mgI} / \mathrm{mL}$ of nonionic contrast medium and a maximum dose of $150 \mathrm{~mL}$ per patient. The warmed contrast medium was administered intravenously with a mechanical power injector at $2.5-5 \mathrm{~mL} / \mathrm{s}$ with a fixed injection duration of $30 \mathrm{~s}$ through a $20-\mathrm{G}$ catheter inserted into an arm vein. Unenhanced, arterial, portal venous, and equilibrium phase images were obtained immediately prior to and 30-45, 65-80, and 180-205 $\mathrm{s}$, respectively, after the start of the contrast material injection [16]. To determine the acquisition delay for hepatic arterial dominant phase imaging, a bolus-tracking technique was used. Arterial phase acquisition was started $22 \mathrm{~s}$ after the trigger threshold (50 HU elevation) was reached at the suprarenal abdominal aorta.

\section{Magnetic Resonance Imaging}

MR images were acquired with 1.5-T or 3-T systems (Table 2). Unenhanced, arterial, portal venous, late, and hepatobiliary phase images were obtained immediately prior to and 25-35, 70-80, $180 \mathrm{~s}$ and 15-20 min, respectively, after bolus injection of $0.025 \mathrm{mmol} / \mathrm{kg}$ body weight $(0.1 \mathrm{~mL} / \mathrm{kg}) \mathrm{Gd}-\mathrm{EOB}-\mathrm{DTPA}$ (Primovist $^{\circledR}$, Bayer-Schering Pharma, Osaka Japan) at a rate of 1-2 mL/s, using a T1-weighted threedimensional gradient-echo sequences in a single breath hold (18-25 s) (LAVA; GE Healthcare, THRIVE; Philips Medical Systems) [9, 12, 16]. A 30-mL saline flush was administered at 1-2 mL/s after Gd-EOBDTPA injection. T2-weighted images were also acquired $[9,12,16]$. Acquisition parameters are listed in Table 2 . 
Imai et al:: Prospective Comparison of Gd-EOB-DTPA-Enhanced MRI with Dynamic CT for Detecting Recurrence of HCC after Radiofrequency Ablation

Table 2. MRI sequences and parameters

\begin{tabular}{|c|c|c|c|c|}
\hline & $\begin{array}{l}\text { Discovery750 }{ }^{\circledR} \\
3.0 \mathrm{~T} \\
\text { (GE Healthcare) }\end{array}$ & $\begin{array}{l}\text { Intera Achieva Nova }{ }^{\circledR} \\
1.5 \mathrm{~T} \text { (Philips Medical } \\
\text { Systems) }\end{array}$ & $\begin{array}{l}\text { Signa Excite HD }{ }^{\circledR} \\
1.5 \mathrm{~T} \\
\text { (GE Healthcare) }\end{array}$ & $\begin{array}{l}\text { Signa Excite HD } \\
3.0 \mathrm{~T} \\
\text { (GE Healthcare) }\end{array}$ \\
\hline \multicolumn{5}{|c|}{ Dynamic, hepatobiliary phase } \\
\hline Sequence & LAVA & THRIVE & LAVA & LAVA \\
\hline $\mathrm{TR}, \mathrm{ms}$ & 3.8 & 4.4 & 4.5 & 3.9 \\
\hline $\mathrm{TE}, \mathrm{ms}$ & 1.8 & 2.1 & 2.2 & 1.7 \\
\hline Flip angle, ${ }^{\circ}$ & 12 & 10 & 12 & 12 \\
\hline Thickness, mm & 4 & 4 & 5 & 4 \\
\hline $\mathrm{FOV}, \mathrm{mm}$ & $288 \times 360$ & 375 & 360 & $340 \times 393$ \\
\hline Matrix & $320 \times 224$ & $192 \times 192$ & $320 \times 192$ & $320 \times 192$ \\
\hline \multicolumn{5}{|c|}{ T2-weighted image } \\
\hline $\mathrm{TR}, \mathrm{ms}$ & 12,000 & $1,502-2,000$ & $-*$ & $4,600-8,500$ \\
\hline $\mathrm{TE}, \mathrm{ms}$ & 70 & 70 & 90 & 91 \\
\hline Thickness, mm & 7 & 7 & 7 & 5 \\
\hline FOV, mm & $320 \times 360$ & 375 & 360 & $340 \times 393$ \\
\hline Matrix & $352 \times 224$ & $256^{\prime} \times 256$ & $256^{\prime} \times 224$ & $512 \times 160$ \\
\hline
\end{tabular}

TR, repetition time; TE, echo time; FOV, field of view; WI, weighted image; LAVA, liver acquisition with volume acceleration; THRIVE, T1 high-resolution isotropic volume excitation; RT, respiratory triggering. * Single-shot fast spin-echo sequencing was used.

\section{Contrast-Enhanced Ultrasonography}

CEUS with Sonazoid ${ }^{\circledR}$ (Daiichi Sankyo, Tokyo, Japan) was carried out as described previously [12] using the coded phase inversion mode with a mechanical index of $0.2-0.3$. The examination consisted of an early vascular phase, a late vascular phase, and a postvascular phase (10-20 min after the injection of the contrast agent).

CT during Hepatic Arteriography/CT during Arterial Portography

Both CTAP and CTHA were performed using a 64-channel MDCT as described previously [16].

\section{Statistical Analysis}

Sensitivity, specificity, positive and negative predictive values, and diagnostic accuracy of Gd-EOBDTPA-enhanced MRI and dynamic MDCT for hypervascular HCC were calculated and were expressed with a 95\% confidence interval. The McNemar test and Fisher's exact probability test were used for statistical comparison of sensitivity, specificity, and accuracy, and of positive and negative predictive values, respectively. Receiver operating characteristic (ROC) analysis was performed to evaluate diagnostic performance in relation to recurrence of hypervascular HCC after RFA. An ROC curve was fitted to each observer's patientbased confidence rating by using a maximum likelihood estimation program (OR-DBM-MRMC ver. 2.5, University of Iowa, http://perception.radiology.uiowa.edu/). The diagnostic performance was then estimated by calculating the area under the ROC curve (AUROC). The univariate $z$ score was calculated to evaluate the significance of the difference between the AUROC values. Alternative free-response ROC (AFROC) analysis (OR-DBM-MRMC ver. 2.5) was performed on a lesion-by-lesion basis for the evaluation of diagnostic performance of recurrence of hypervascular HCC after RFA [16]. The area under each AFROC curve (AUROC) was used to indicate the overall diagnostic performance of each technique and each observer. Regarding the 4-point scale used to evaluate diagnostic performance, interobserver agreement between the 2 observers was determined by the unweighted $\kappa$ statistic [16]. For all statistical analyses other than ROC analysis, a commercial software package (SPSS for Windows, version $17.0^{\circledR}$; SPSS, Chicago, IL, USA) was used. A twotailed $p$ value $<0.05$ was considered to indicate a significant difference. Power analysis indicated that a minimum sample size of 100 patients would provide $80 \%$ power and a $5 \%$ significance level in the detection of a mean difference of $30 \%$ between values of Gd-EOB-DTPA-enhanced MRI and dynamic MDCT. 


\section{Liver \\ Cancer}
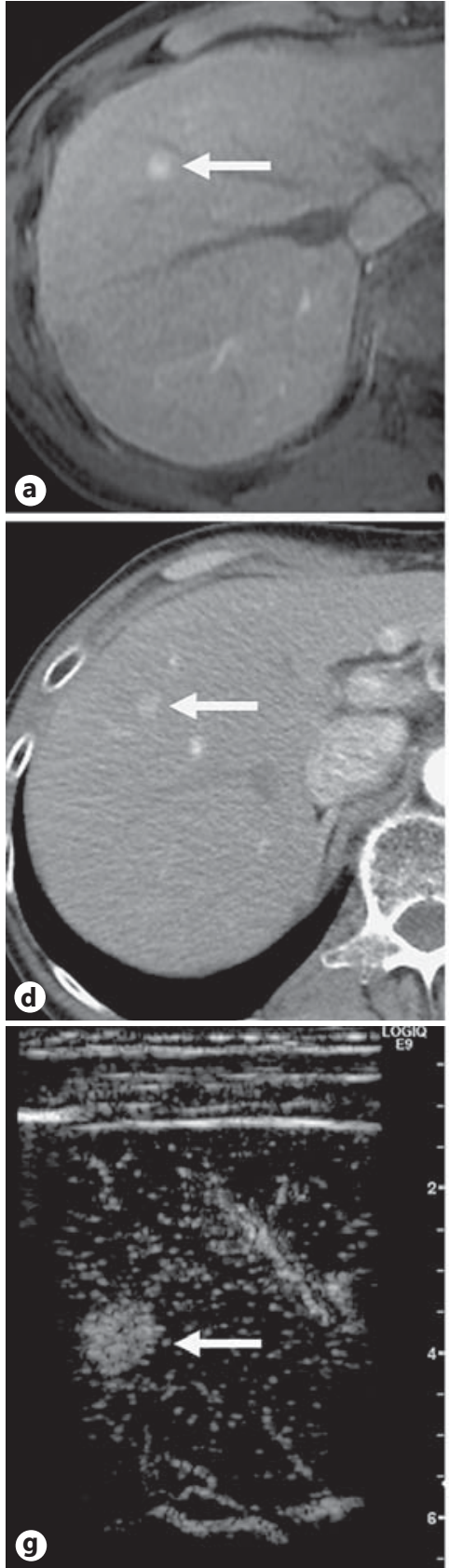

\begin{tabular}{l|l}
\hline Liver Cancer 2017;6:349-359 \\
\hline DOI: 10.1159/000481416 & $\begin{array}{l}\text { ○ 2017 S. Karger AG, Basel } \\
\text { www.karger.com/lic }\end{array}$ \\
\hline
\end{tabular}

Imai et al.: Prospective Comparison of Gd-EOB-DTPA-Enhanced MRI with Dynamic CT for Detecting Recurrence of HCC after Radiofrequency Ablation
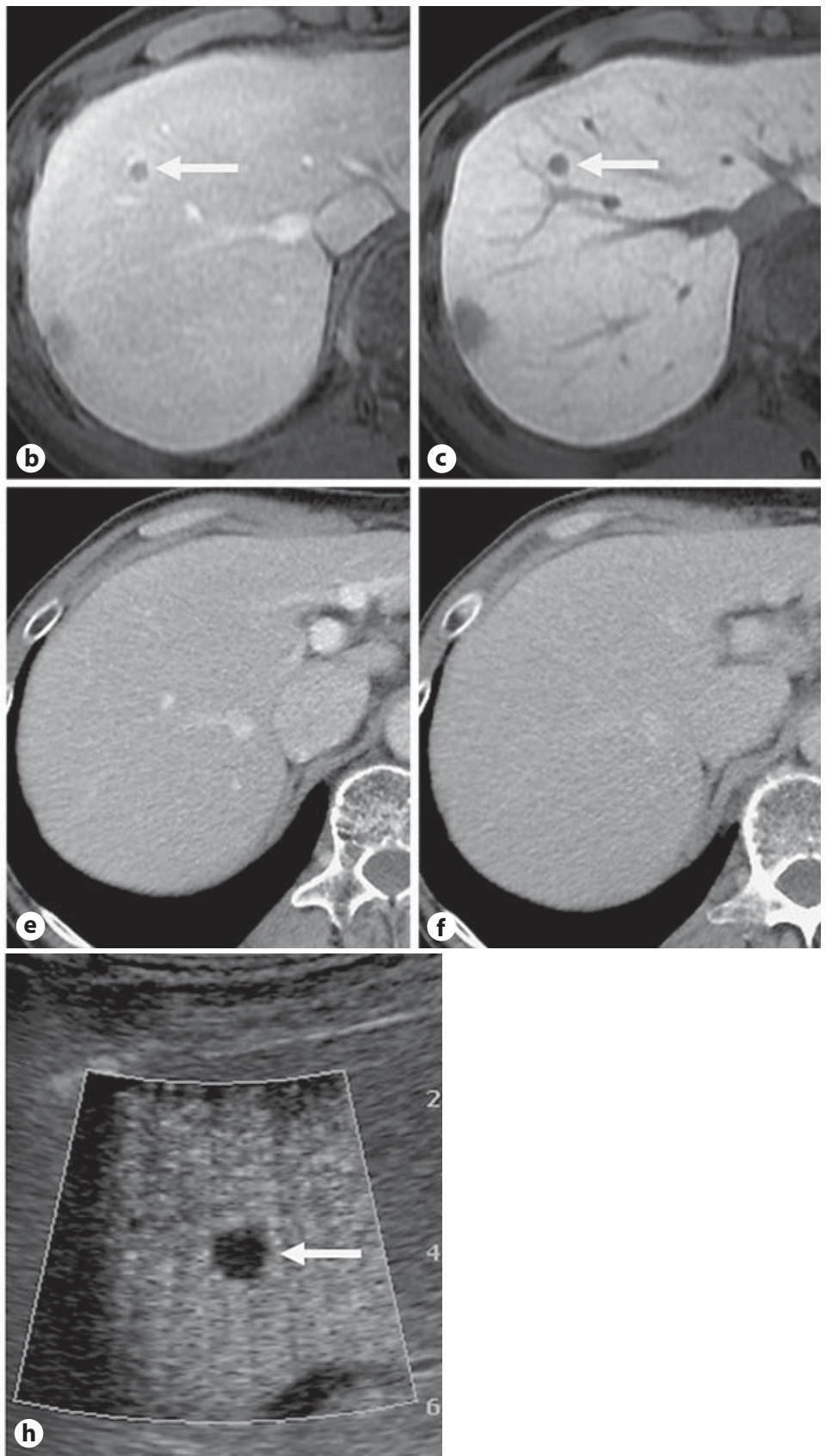

Fig. 1. Images in a 55-year-old man with chronic hepatitis B developing recurrence of hypervascular HCC 9 months after curative treatment of HCC with RFA. The tumor (arrow) in segment 8, $0.8 \mathrm{~cm}$ in diameter, is hyperintense on the arterial phase (a) and hypointense on the portal venous phase (b) and hepatobiliary phase (c) of Gd-EOB-DTPA-enhanced MRI. The corresponding multiphasic CT image obtained during the arterial phase (d) shows a hypervascular nodule, but the nodule shows isoattenuation on the portal venous (e) and equilibrium (f) phase images. Contrast-enhanced ultrasonography with Sonazoid ${ }^{\circledR}$ shows a hypervascular nodule on the early vascular phase image $(\mathbf{g})$ and a clear perfusion defect on the postvascular phase image (h). 
Imai et al.: Prospective Comparison of Gd-EOB-DTPA-Enhanced MRI with Dynamic CT for Detecting Recurrence of HCC after Radiofrequency Ablation

Table 3. Patient-based diagnostic performance of Gd-EOB-DTPA-enhanced MRI and dynamic MDCT for recurrence of hypervascular HCC in 97 patients

\begin{tabular}{|c|c|c|c|c|c|c|c|}
\hline & \multicolumn{3}{|c|}{ Gd-EOB-DTPA-enhanced MRI } & \multicolumn{3}{|c|}{ Dynamic MDCT } & \multirow[t]{2}{*}{$p$ value } \\
\hline & & patients, $n$ & $95 \% \mathrm{CI}$ & & patients, $n$ & $95 \% \mathrm{CI}$ & \\
\hline \multicolumn{8}{|l|}{ Observer 1} \\
\hline AUROC & 0.95 & & $0.91-0.99$ & 0.76 & & $0.67-0.84$ & $<0.001^{\mathrm{a}}$ \\
\hline Sensitivity & 0.88 & $42 / 48$ & $0.78-0.97$ & 0.52 & $25 / 48$ & $0.37-0.67$ & $<0.001^{\mathrm{b}}$ \\
\hline Specificity & 0.94 & $46 / 49$ & $0.87-1.01$ & 0.92 & $45 / 49$ & $0.84-1.00$ & $1^{\mathrm{b}}$ \\
\hline Accuracy & 0.91 & $88 / 97$ & $0.85-0.97$ & 0.72 & $70 / 97$ & $0.63-0.81$ & $0.002^{\mathrm{b}}$ \\
\hline PPV & 0.93 & $42 / 45$ & $0.86-1.01$ & 0.86 & $25 / 29$ & $0.73-1.00$ & $0.27^{\mathrm{c}}$ \\
\hline NPV & 0.88 & $46 / 52$ & $0.79-0.97$ & 0.66 & $45 / 68$ & $0.55-0.78$ & $0.004^{c}$ \\
\hline \multicolumn{8}{|l|}{ Observer 2} \\
\hline AUROC & 0.90 & & $0.84-0.96$ & 0.74 & & $0.66-0.83$ & $0.002^{\mathrm{a}}$ \\
\hline Sensitivity & 0.83 & $40 / 48$ & $0.72-0.94$ & 0.52 & $25 / 48$ & $0.37-0.67$ & $<0.001^{b}$ \\
\hline Specificity & 0.94 & $46 / 49$ & $0.87-1.01$ & 0.92 & $45 / 49$ & $0.84-1.00$ & $1^{\mathrm{b}}$ \\
\hline Accuracy & 0.89 & 86/97 & $0.82-0.95$ & 0.72 & $70 / 97$ & $0.63-0.81$ & $0.004^{\mathrm{b}}$ \\
\hline PPV & 0.93 & $40 / 43$ & $0.85-1.01$ & 0.86 & $25 / 29$ & $0.73-1.00$ & $0.29^{c}$ \\
\hline NPV & 0.85 & $46 / 54$ & $0.75-0.95$ & 0.66 & $45 / 68$ & $0.55-0.78$ & $0.013^{c}$ \\
\hline
\end{tabular}

AUROC, area under the receiver operating characteristic curve; PPV, positive predictive value; NPV, negative predictive value. ${ }^{a}$ Univariate $z$ score test. ${ }^{b}$ McNemar test. ${ }^{c}$ Fisher's exact probability test.

\section{Results}

Development of Hypervascular HCC

During the follow-up period, recurrent HCCs with the typical vascular pattern diagnosed by on-site consensus reading were observed in 48 of 97 patients. Of the 48 patients, 22 were diagnosed as having HCC by both Gd-EOB-DTPA-enhanced MRI and dynamic MDCT, 22 only by Gd-EOB-DTPA-enhanced MRI combined with CEUS or CTHA/CTAP, and 4 only by dynamic MDCT combined with CEUS or CTHA/CTAP. Consequently, recurrence of HCC was diagnosed in more patients by Gd-EOB-DTPA-enhanced MRI than by dynamic MDCT (44 patients, $91.7 \%$, vs. 26 patients, $54.2 \%$, respectively, $p<0.001$ ).

In the 48 patients with recurrence, $66 \mathrm{HCCs}$ with a typical vascular pattern were detected. The median tumor size was $9 \mathrm{~mm}$ (range, 5-20). Of the 66 HCCs, 27 HCCs were diagnosed by both Gd-EOB-DTPA-enhanced MRI and dynamic MDCT, 32 only by Gd-EOB-DTPA-enhanced MRI combined with CEUS or CTHA/CTAP (Fig. 1), and 7 only by dynamic MDCT combined with CEUS or CTHA/CTAP. Thus, more HCCs were diagnosed by Gd-EOB-DTPA-enhanced MRI than by dynamic MDCT (59 HCCs, $89.4 \%$, vs. 34 HCCs, $51.5 \%$, respectively, $p<0.001$ ).

\section{Diagnostic Performance Analysis of Hypervascular HCC by Blinded Observers}

AUROC values, accuracy, sensitivity, specificity, and positive predictive and negative predictive values for the recurrence of hypervascular HCCs of 2 observers on a patient-based analysis in 97 patients are shown in Table 3. The AUROC values of the patient-based ROC analysis of Gd-EOB-DTPA-enhanced MRI (observer 1: 0.95, observer 2: 0.90) were significantly higher than those of MDCT (observer 1: 0.76, observer 2: 0.74) (Table 3). Sensitivity, accuracy, and negative predictive values of MRI were also significantly higher than those of MDCT in both observers. There were no differences in specificity and positive predictive value between the observers. 
Table 4. A tumor-by-tumor diagnostic performance of Gd-EOB-DTPA-enhanced MRI and multiphasic multidetector CT for hypervascular HCC $(n=66)$

\begin{tabular}{|c|c|c|c|c|c|c|c|c|}
\hline & & \multicolumn{3}{|c|}{ Gd-EOB-DTPA-enhanced MRI } & \multicolumn{3}{|c|}{ Multiphasic CT } & \multirow[t]{2}{*}{$p$ value } \\
\hline & & & tumors, $n$ & $95 \% \mathrm{CI}$ & & tumors, $n$ & $95 \% \mathrm{CI}$ & \\
\hline \multicolumn{9}{|l|}{ Observer 1} \\
\hline \multirow{2}{*}{$\begin{array}{l}\text { All lesions } \\
\qquad(n=66)\end{array}$} & AUROC & 0.88 & & $0.83-0.93$ & 0.69 & & $0.62-0.76$ & $<0.001^{\mathrm{a}}$ \\
\hline & Sensitivity & 0.76 & $50 / 66$ & $0.65-0.86$ & 0.42 & $28 / 66$ & $0.30-0.55$ & $<0.001^{\mathrm{b}}$ \\
\hline \multirow{2}{*}{$\begin{array}{l}\text { Lesions }<1 \mathrm{~cm} \\
\qquad(n=35)\end{array}$} & AUROC & 0.90 & & $0.83-0.96$ & 0.72 & & $0.62-0.81$ & $0.002^{\mathrm{a}}$ \\
\hline & Sensitivity & 0.74 & $26 / 35$ & $0.59-0.90$ & 0.37 & $13 / 35$ & $0.20-0.54$ & $0.011^{b}$ \\
\hline \multirow{2}{*}{$\begin{array}{l}\text { Lesions } \geq 1 \mathrm{~cm} \\
\qquad(n=31)\end{array}$} & AUROC & 0.90 & & $0.83-0.97$ & 0.74 & & $0.65-0.84$ & $0.006^{\mathrm{a}}$ \\
\hline & Sensitivity & 0.77 & $24 / 31$ & $0.62-0.93$ & 0.48 & $15 / 31$ & $0.30-0.67$ & $0.049^{b}$ \\
\hline \multicolumn{9}{|l|}{ Observer 2} \\
\hline \multirow{2}{*}{$\begin{array}{l}\text { All lesions } \\
\qquad(n=66)\end{array}$} & AUROC & 0.85 & & $0.79-0.91$ & 0.71 & & $0.64-0.78$ & $0.002^{\mathrm{a}}$ \\
\hline & Sensitivity & 0.76 & $50 / 66$ & $0.65-0.86$ & 0.44 & $29 / 66$ & $0.31-0.56$ & $<0.001^{\mathrm{b}}$ \\
\hline \multirow{2}{*}{$\begin{array}{l}\text { Lesions }<1 \mathrm{~cm} \\
\qquad(n=35)\end{array}$} & AUROC & 0.84 & & $0.76-0.93$ & 0.71 & & $0.62-0.81$ & $0.024^{\mathrm{a}}$ \\
\hline & Sensitivity & 0.74 & $26 / 35$ & $0.59-0.90$ & 0.43 & $15 / 35$ & $0.26-0.60$ & $0.013^{b}$ \\
\hline \multirow{2}{*}{$\begin{array}{l}\text { Lesions } \geq 1 \mathrm{~cm} \\
\qquad(n=31)\end{array}$} & AUROC & 0.90 & & $0.83-0.97$ & 0.75 & & $0.65-0.85$ & $0.015^{\mathrm{a}}$ \\
\hline & Sensitivity & 0.77 & $24 / 31$ & $0.62-0.93$ & 0.45 & $14 / 35$ & $0.27-0.64$ & $0.031^{b}$ \\
\hline
\end{tabular}

AUROC, area under the alternative free-response receiver operating characteristic curve. ${ }^{\text {a }}$ Univariate $z$ score test. ${ }^{\mathrm{b}}$ McNemar test.

AFROC analysis on a tumor-by-tumor basis revealed that the AUROC value of Gd-EOBDTPA-enhanced MRI (observer 1: 0.88, observer 2: 0.85) were significantly higher than those of MDCT (observer 1: 0.69, observer 2: 0.71) (Table 4). Sensitivities of the tumor-by-tumor analysis of MRI (observer 1: 0.76, observer 2: 0.76 ) were also significantly higher than those of MDCT (observer 1: 0.42 , observer 2: 0.44 ) (Table 4). For lesions $<1 \mathrm{~cm}$ in diameter $(n=35)$ and those $\geq 1 \mathrm{~cm}$ in diameter $(n=31)$, AUROC values and sensitivities of MRI were also significantly higher than those of MDCT in both observers (Table 4).

As for the patient-based analysis, interobserver agreement was excellent for Gd-EOB-

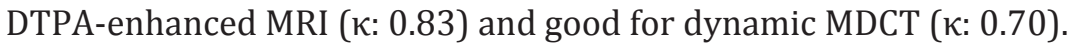

\section{BCLC Stage at Recurrence}

The 4 patients with a hypovascular nodule were diagnosed with a well-differentiated HCC on biopsy. Table 5 shows BCLC stages evaluated by dynamic MDCT, Gd-EOB-DTPAenhanced MRI, or the combination of both modalities in 52 patients with recurrence of typical hypervascular HCC or hypovascular HCC. Forty-eight of the 52 patients were diagnosed as having HCC based on Gd-EOB-DTPA-enhanced MRI evaluation, 47 of which were categorized as BCLC stage 0 or A.

\section{Discussion}

We have clearly demonstrated that Gd-EOB-DTPA-enhanced MRI showed significantly higher diagnostic accuracy and sensitivity in the detection of recurrent HCC after RFA compared with dynamic MDCT. More HCCs were diagnosed by Gd-EOB-DTPA-enhanced MRI than by dynamic MDCT by on-site consensus reading in this prospective multicenter study. In addition to the on-site consensus reading, we also performed both patient-based and 
Imai et al:: Prospective Comparison of Gd-EOB-DTPA-Enhanced MRI with Dynamic CT for Detecting Recurrence of HCC after Radiofrequency Ablation

Table 5. BCLC stage evaluated by dynamic MDCT, Gd-EOB-DTPA-enhanced MRI, or either in 52 patients with recurrence of hypervascular $(n=48)$ or hypovascular $(n=4)$ HCC

\begin{tabular}{lllll}
\hline Modality for evaluation of staging & \multicolumn{2}{l}{ BCLC stage } & \multicolumn{2}{c}{$\begin{array}{c}\text { HCC not } \\
\text { detected }^{\mathrm{a}}\end{array}$} \\
\cline { 2 - 4 } & 0 & A & B & \\
\hline Dynamic MDCT & $21(40)$ & $7(13)$ & 0 & $24(46)$ \\
Gd-EOB-DTPA-enhanced MRI & $39(75)$ & $8(15)$ & $1(2)$ & $4(8)$ \\
Dynamic MDCT or Gd-EOB-DTPA-enhanced MRI & $41(79)$ & $10(19)$ & $1(2)$ & 0 \\
\hline
\end{tabular}

Values indicate patient number (\%). BCLC, Barcelona Clinic Liver Cancer. ${ }^{a}$ Of the 52 patients with recurrence, HCC was not detected in 24 and 4 patients by dynamic MDCT and Gd-EOB-DTPA-enhanced MRI, respectively. ${ }^{\mathrm{b}}$ HCC was detected by either dynamic MDCT or Gd-EOB-DTPA-enhanced MRI.

lesion-based diagnostic performance analyses. In the patient-based analysis, the accuracy, sensitivity, negative predictive value and AUROC of Gd-EOB-DTPA-enhanced MRI were significantly higher than those of dynamic MDCT, and interobserver agreements were excellent for Gd-EOB-DTPA-enhanced MRI and good for dynamic CT. Also, in the lesion-based analysis, the sensitivity and AUROC of Gd-EOB-DTPA-enhanced MRI were significantly higher than those of multiphasic MDCT. These results suggest that Gd-EOB-DTPA-enhanced MRI is preferable to dynamic MDCT for follow-up of patients with HCC treated with RFA.

There have been several studies comparing the diagnostic performance of Gd-EOBDTPA-enhanced MRI and dynamic MDCT. Kim et al. [24] reported that Gd-EOB-DTPAenhanced MRI had diagnostic performance similar to dynamic MDCT in patients with relatively large tumor sizes with a median size of $2.9 \mathrm{~cm}$ (range, $0.5-10.5 \mathrm{~cm}$ ), while MRI might be better than dynamic MDCT in the detection of HCC $\leq 1 \mathrm{~cm}$ in diameter. Hwang et al. [14] retrospectively compared the diagnostic performance of Gd-EOB-DTPA-enhanced MRI with that of dynamic MDCT to evaluate recurrence after transcatheter arterial chemoembolization or RFA. They found that Gd-EOB-DTPA-enhanced MRI had better diagnostic performance than dynamic MDCT for evaluating the recurrence following either treatment. These results are compatible with our results, although their study was retrospective and had inherent selection bias. Di Martino et al. [13] also reported that compared with 64-detector dynamic MDCT, Gd-EOB-DTPA-enhanced MRI yielded significantly higher diagnostic accuracy and sensitivity in the detection of HCC in cirrhotic patients.

Recent studies have reported that additional Gd-EOB-DTPA-enhanced MRI examinations found new intrahepatic HCC lesions in patients with BCLC stage 0 or A based on the diagnosis by conventional dynamic CT $[20,21]$. Moreover, additional evaluation by Gd-EOB-DTPA-enhanced MRI in the patients with a single HCC initially diagnosed by dynamic CT resulted in improved recurrence-free and overall survival [21]. However, these studies were retrospectively conducted, and dynamic CT examination was performed as a first survey for HCC, followed by Gd-EOB-DTPA-enhanced MRI. Accordingly, Gd-EOBDTPA-enhanced MRI was never used as the first imaging modality for detecting HCC. As a result, the median size of HCC in the report by Kim et al. [21] $(2.7 \mathrm{~cm})$ was much larger than the size in our study $(0.9 \mathrm{~cm})$. To the best of our knowledge, our study is the first prospective study investigating the efficacy of Gd-EOB-DTPA-enhanced MRI for the evaluation of recurrence after RFA for HCC.Gd-EOB-DTPA-enhanced MRI diagnosed the majority of HCC recurrences at BCLC stage 0 or A (Table 5 ) and their diameters were $\leq 2 \mathrm{~cm}$, suggesting that the earlier detection of smaller recurrences following curative treatment with RFA was enabled by the high diagnostic performance of Gd-EOB-DTPA-enhanced MRI 
in our study protocol, or that the recurrence rate in our study population was unusually high.

Our study has some limitations. First, diagnosis of HCC at the time of recurrence was not made pathologically in most cases because it was difficult to obtain a biopsy specimen from the ethical point of view. Instead, diagnosis of HCC was made based on the typical vascular pattern on 2 imaging modalities by the consensus of experienced radiologists and hepatologists. Second, this study was conducted with a single-arm study design since the purpose of the study was to prospectively compare the efficacy of Gd-EOB-DTPA-enhanced MRI with that of dynamic MDCT in the detection of recurrent of HCC. Third, we examined a relatively small number of patients, although we calculated the sample size of this study by power analysis.

In conclusion, compared with dynamic MDCT, Gd-EOB-DTPA-enhanced MRI had greater diagnostic performance for detection of HCC recurrence and may be a better tool for following patients after RFA.

\section{Acknowledgments}

We thank Dr. Yoshiyuki Sawai, Dr. Hideki Hagihara, Dr. Masahide Oshita, and Dr. Hideki Ishikawa for their great contribution in the discussion about study design.

\section{Disclosure Statement}

This study was funded by Bayer Yakuhin, Ltd. The funder had no role in the study design, data collection and analysis, preparation of the manuscript, or decision of publication. The authors declare no further conflict of interest.

\section{Author Contributions}

All authors: approval of the final version of the manuscript. Y.I.: study concept and design, interpretation of data, drafting of the manuscript, and study supervision. K.K.: study concept and design, interpretation of data, and critical revision of the manuscript. T.M., T.T.: study concept and design. M.H.: statistical analysis and critical revision of the manuscript. T.Y., K.F., T. Itoh, T. Igura, M.S., M. Takamura, K.N., N.U., K.T., T.K.: acquisition of clinical data and critical revision of the manuscript. M. Tsurusaki, H.T., H.O.: analysis of data.

\section{References}

1 Jemal A, Bray F, Center MM, Ferlay J, Ward E, Forman D: Global cancer statistics. CA Cancer J Clin 2011;61: 69-90.

2 Bosch FX, Ribes J, Díaz M, Cléries R: Primary liver cancer: worldwide incidence and trends. Gastroenterology 2004;127:S5-S16.

3 Kanwal F, Hoang T, Kramer JR, Asch SM, Goetz MB, Zeringue A, Richardson P, El-Serag HB: Increasing prevalence of HCC and cirrhosis in patients with chronic hepatitis C virus infection. Gastroenterology 2011;140: $1182-1188$.

4 Kudo M: Surveillance, diagnosis, treatment, and outcome of liver cancer in Japan. Liver Cancer 2015;4:39-50.

5 Bruix J, Sherman M; Practice Guidelines Committee, American Association for the Study of Liver Diseases: Management of hepatocellular carcinoma. Hepatology 2005;42:1208-1236.

6 European Association for the Study of the Liver, European Organisation for Research and Treatment of Cancer: EASL-EORTC Clinical Practice Guidelines: Management of Hepatocellular Carcinoma. J Hepatol 2012;56:908943.

7 Toyoda H, Kumada T, Tada T, Niinomi T, Ito T, Sone Y, Kaneoka Y, Maeda A: Non-hypervascular hypointense nodules detected by Gd-EOB-DTPA-enhanced MRI are a risk factor for recurrence of HCC after hepatectomy. J Hepatol 2013;58:1174-1180. 
Imai et al.: Prospective Comparison of Gd-EOB-DTPA-Enhanced MRI with Dynamic CT for Detecting Recurrence of HCC after Radiofrequency Ablation

8 Lee DH, Lee JM, Lee JY, Kim SH, Kim JH, Yoon JH, Kim YJ, Lee JH, Yu SJ, Han JK, Choi BI: Non-hypervascular hepatobiliary phase hypointense nodules on gadoxetic acid-enhanced MRI: risk of HCC recurrence after radiofrequency ablation. J Hepatol 2015;62:1122-1130.

9 Kogita S, Imai Y, Okada M, Kim T, Onishi H, Takamura M, Fukuda K, Igura T, Sawai Y, Morimoto O, Hori M, Nagano H, Wakasa K, Hayashi N, Murakami T: Gd-EOB-DTPA-enhanced magnetic resonance images of hepatocellular carcinoma: correlation with histological grading and portal blood flow. Eur Radiol 2010;20:24052413.

10 Sano K, Ichikawa T, Motosugi U, Sou H, Muhi AM, Matsuda M, Nakano M, Sakamoto M, Nakazawa T, Asakawa M, Fujii H, Kitamura T, Enomoto N, Araki T: Imaging study of early hepatocellular carcinoma: usefulness of gadoxetic acid-enhanced MR imaging. Radiology 2011;261:834-844.

11 Kitao A, Matsui O, Yoneda N, Kozaka K, Shinmura R, Koda W, Kobayashi S, Gabata T, Zen Y, Yamashita T, Kaneko S, Nakanuma Y: The uptake transporter OATP8 expression decreases during multistep hepatocarcinogenesis: correlation with gadoxetic acid enhanced MR imaging. Eur Radiol 2011;21:2056-2066.

12 Ohama H, Imai Y, Nakashima O, Kogita S, Takamura M, Hori M, Seki Y, Sawai Y, Igura T, Fukuda K, Makino Y, Morimoto O, Ohsawa M, Sakamoto M, Murakami T: Images of Sonazoid-enhanced ultrasonography in multistep hepatocarcinogenesis: comparison with Gd-EOB-DTPA-enhanced MRI. J Gastroenterol 2014;49:1081-1093.

13 Di Martino M, Marin D, Guerrisi A, Baski M, Galati F, Rossi M, Brozzetti S, Masciangelo R, Passariello R, Catalano C: Intraindividual comparison of gadoxetate disodium-enhanced MR imaging and 64-section multidetector CT in the detection of hepatocellular carcinoma in patients with cirrhosis. Radiology 2010;256:806-816.

14 Hwang J, Kim SH, Kim YS, Lee MW, Woo JY, Lee WJ, Lim HK: Gadoxetic acid-enhanced MRI versus multiphase multidetector row computed tomography for evaluating the viable tumor of hepatocellular carcinomas treated with image-guided tumor therapy. J Magn Reson Imaging 2010;32:629-38.

15 Haradome H, Grazioli L, Tinti R, Morone M, Motosugi U, Sano K, Ichikawa T, Kwee TC, Colagrande S: Additional value of gadoxetic acid-DTPA-enhanced hepatobiliary phase MR imaging in the diagnosis of early-stage hepatocellular carcinoma: comparison with dynamic triple-phase multidetector CT imaging. J Magn Reson Imaging 2011;34:69-78.

16 Onishi H, Kim T, Imai Y, Hori M, Nagano H, Nakaya Y, Tsuboyama T, Nakamoto A, Tatsumi M, Kumano S, Okada M, Takamura M, Wakasa K, Tomiyama N, Murakami T: Hypervascular hepatocellular carcinomas: detection with gadoxetate disodium-enhanced MR imaging and multiphasic multidetector CT. Eur Radiol 2012;22:845854.

17 Tsurusaki M, Sofue K, Isoda H, Okada M, Kitajima K, Murakami T: Comparison of gadoxetic acid-enhanced magnetic resonance imaging and contrast-enhanced computed tomography with histopathological examinations for the identification of hepatocellular carcinoma: a multicenter phase III study. J Gastroenterol 2016: 51:71-79.

18 Lee YJ, Lee JM, Lee JS, Lee HY, Park BH, Kim YH, Han JK, Choi BI: Hepatocellular carcinoma: diagnostic performance of multidetector CT and MR imaging - a systematic review and meta-analysis. Radiology 2015;275: 97-109.

19 Van Beers BE, Pastor CM, Hussain HK: Primovist, Eovist: what to expect? J Hepatol 2012;57:421-429.

20 Jin YJ, Nah SY, Lee JW, Lee JI, Jeong S, Lee DH, Kim YS, Cho SG, Jeon YS: Utility of adding Primovist magnetic resonance imaging to analysis of hepatocellular carcinoma by liver dynamic computed tomography. Clin Gastroenterol Hepatol 2013;11:187-192.

21 Kim HD, Lim YS, Han S, An J, Kim GA, Kim SY, Lee SJ, Won HJ, Byun JH: Evaluation of early-stage hepatocellular carcinoma by magnetic resonance imaging with gadoxetic acid detects additional lesions and increases overall survival. Gastroenterology 2015;148:1371-1382.

22 Sakamoto M, Hirohashi S, Shimosato Y: Early stages of multistep hepatocarcinogenesis: adenomatous hyperplasia and early hepatocellular carcinoma. Hum Pathol 1991;22:172-178.

23 The International Consensus Group for Hepatocellular Neoplasia: Pathologic diagnosis of early hepatocellular carcinoma: a report of the international consensus group for hepatocellular neoplasia. Hepatology 2009;49: 658-664.

24 Kim YK, Kim CS, Chung GH, Han YM, Lee SY, Chon SB, Lee JM: Comparison of gadobenate dimeglumineenhanced dynamic MRI and 16-MDCT for the detection of hepatocellular carcinoma. AJR Am J Roentgenol 2006;186:149-157. 